\title{
Retraining of dysgraphia - a case study
}

\author{
N. KAPUR AND D. S. GORDON \\ From the Neurosurgical Unit, Royal Victoria Hospital, Belfast
}

SYNOPSIS A patient with dysgraphia resulting from a gunshot wound of the brain is presented. Analysis of the functional status of component operations involved in the skill of writing is described. A retraining scheme based on this analysis brought about a significant improvement in the patient's handwriting.

The purpose of this note is to discuss a case of dysgraphia, the investigative methods which were employed, and the retraining scheme by which an improvement in writing performance was produced.

Dysgraphia has usually been regarded as particularly insensitive to training procedures. Butfield and Zangwill (1946) noted: 'In patients who had lost the capacity to form letters as such (whether as a primary agraphia or as part of a constructional apraxia), no satisfactory method of re-education was discovered' (p. 77). Since then, little systematic work on dysgraphia and its retraining has been carried out, with the exception of the writings by Russian workers (Luria et al., 1969), who made this summary of the available knowledge: 'Few attempts have been made to make a special neuropsychological analysis of the process of writing and disturbances of writing in local brain lesions are interpreted relatively superficially in the neuropsychological literature' (p. 382). Of the possible reasons for this lack of knowledge, two may be mentioned: firstly, disturbances in speech production often interact with writing disorders and, being more readily noticeable and more immediately significant for everyday adaptation, are more likely to receive attention by research workers and therapists alike. Secondly, difficulties in writing usually result from lesions of the left hemisphere; these lesions are often accompanied by a right mono- or herniparesis. Analysis of the writing disturbance may then be made practically impossible because of inability to perform any manual skills on the right side.

(Accepted 30 November 1974.)
In the case to be reported, dysgraphia was more amenable to analysis since there was little impairment of speech production and no weakness of the right hand.

\section{CASE REPORT}

The patient, a man aged 24 years, was admitted to the Department of Neurosurgery, Royal Victoria Hospital, Belfast, in August 1972 with a gunshot wound of the head. On admission, there was a large scalp defect, measuring $7.5 \times 5 \mathrm{~cm}$, in the left posterior parietal area which was bleeding profusely. Radiography of the skull showed an extensive comminuted fracture and revealed numerous shotgun pellets in the scalp and brain. Extensive debridement was required. There was a long dural tear and extensive brain laceration. The patient remained in hospital for approximately 11 weeks. The patient, who is right-handed, had initial right hemiparesis; some 'word-finding' problems in speech had almost cleared up at the time of discharge, and there was little difficulty in comprehension of speech. His performance on the Wechsler Adult Intelligence Scale was found to be rather below average, particularly on visuospatial tasks. There was considerable reading difficulty, and fluency in writing was limited to his own name.

He was readmitted six months later for cranioplasty. The site and extent of the skull and brain damage is indicated by the area covered by the titanium plate (Fig. 1). He had already started work as a labourer on a building site. The right-sided weakness had now cleared up completely and speech was normal. Reading, however, was still rather laborious and he often gave up after a few lines, depending on the type of material which he was reading. He also complained of some difficulty in 'concentration'. He had the features of the Gerst- 


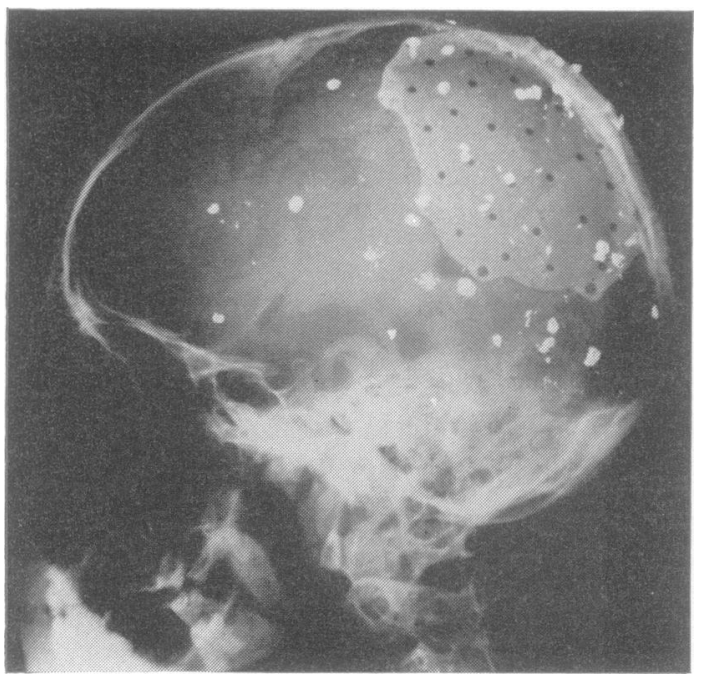

FIG. 1 Extent of left parietal defect in patient as shown by area of titanium plate. Note pieces of shotgun pellet still embedded in the skull.

mann syndrome: left-right disorientation acalculia, finger agnosia, and dysgraphia. The latter disability had shown little recovery in the intervening period of six months. It was characterized by slow and fragmentary writing of words and was investigated in greater depth.

\section{INVESTIGATION}

Luria et al. (1969) have subdivided the skill of writing into five stages - phonetic analysis, articulatory analysis, translation of the phonetic code into a visual code, translation of the visual code into a motor programme, and, lastly, the maintenance of a plan to direct-and, in the event of errors, to correct-particular units of behaviour. Luria's approach was not implemented directly, but a similar analysis was made of the patient's dysgraphia. This involved an examination of each of the component operations in writing, and then developing and executing a training programme to improve the functioning of any defective component operations. In this paper, the term 'phoneme' is used to refer to one of the $\mathbf{3 0}$ to $\mathbf{4 0}$ distinctive sounds in the English language. It is more specific in its connotation than the word 'syllable' which may often refer to a group of phonemes. Thus, the syllable 'bu' consists of the phoneme sounds associated with the letters ' $b$ ' and ' $u$ '. The term 'grapheme' is used to refer to the visual representation or code corresponding to a particular phoneme. It can refer to a group of letters (as in the case of the phoneme 'sh') or to a single letter (as in the sound associated with the letter ' $b$ ').

ANALYSIS OF THE SKILL Non-specific sources of writing difficulty A preliminary investigation was first carried out to search for any general factors which could explain the patient's dysgraphia. Although he left school when aged 15, he said he could write well before he was injured. He did not have a hemianopia or other primary visual upset. Motor function was normal and he did not have parietal lobe sensory disturbances which could have interfered with skilled motor activities. There was also the possibility that, considering his difficulty in reading, there was some general defect in the visual representation of letters of the alphabet; however, the fact that he could successfully read all the letters of the alphabet, yet was unable to write some of the letters, argues against this hypothesis. As noted earlier, the writing difficulty was not secondary to a more general difficulty in language production since his speech was normal for a man from his station in life.

Specific components of writing It should be pointed out that the following analysis of specific components of the dysgraphia refers to externally initiated as opposed to spontaneous or self-initiated writing. AO dysphasic patient may often be unable to repeat $a \leq \overrightarrow{0}$ word which is spoken to him, yet he may be able to윽 use the word quite readily in spontaneous conversa-? tion. This distinction does not apply so much to a less 'automatic' skill such as writing, although it should be borne in mind that in the present case samples of writing were based on letters and words dictated to the patient.

1. Breakdown into phonemic units The first specific component of handwriting which was considered was the ability to break down words into their distinctive phonemic parts. In order to retain accurately the acoustic representation of words, it is necessary not only to retain the distinctive sounds but also their sequence. The intactness of this component operation can be tested simply by asking the patient to repeat a word from dictation. As was expected from his normal conversational ability, our patient performed correctly on this task. The retention of phonemic units may also be impaired while spelling and writing of them is taking place, especially in the case of words containing many syllables. The difficulty may be indicated in the incomplete writing of words. It was clear that this was not the major cause of our patient's writing difficulty since he also had difficulty in writing short words and even single letters. 
2. Analysis into alphabetical units After having identified accurately and retained the phonemic units of a word, the patient must next break down the units into individual letters of the alphabet. Evidence on this ability was obtained by simply asking the patient to spell common words, and he showed little difficulty on this task. Correct performance of component 2 depends, of course, on the successful functioning of component 1 . However, since the reverse does not hold-that is, component 1 may be intact yet component 2 may be defectivethe two operations are presented here as logically distinct.

The translation of each phoneme into its corresponding grapheme requires that the patient must next be able to form a visual representation of each letter. That the patient could in fact correctly perform this component of writing was suggested by his normal reading of individual letters and confirmed by his ability to point out a spoken letter from a series of letters presented before him.

3. Spatial-motor representation of letters The spatial-motor component of writing was tested by asking the patient both to copy letters already written down before him, and to write letters presented acoustically. Although in copying letters he made a few mistakes, his chief difficulty lay in writing from dictation. The patient could manage most upper-case letters but could succeed in writing only 21 lower-case letters, and these with an average latency of nine seconds. It was evident, therefore, that the patient's difficulty lay in retrieving from memory the particular spatial movements which he had to make in order to write each letter. It was next decided to find out if the difficulty in making graphic responses was limited to linguistic codes. If this were the case, the possibility existed that one manifestation of his graphic ability could be used to assist the recovery of another part. However, as might be expected in the case of a left parietal injury, our patient had difficulty in drawing from memory common geometric shapes or common objects.

\section{TREATMENT}

BASIC STRATEGY The basic method of retraining was to use the patient's repertoire of efficient responses to aid the recovery of inefficient response patterns. Within this broad approach, two general techniques were employed-providing the patient with assistance in the performance of writing, with gradual reduction of this support in subsequent training sessions; and, as will be explained in the next section, encouraging the patient to adopt alternative coding strategies in his production of spatial-motor responses. The emphasis in retraining was on the writing of lower-case letters, since they are of more importance for most writing tasks.

PROVISION OF ASSISTANCE The patient's ability to copy was exploited by giving him practice in copying letters and writing over letters; these letters had been written in advance for the patient. This technique was used particularly with letters which he could not write spontaneously. It was important in the case of some letters-for example, ' $r$ '-to find his natural way of writing the letter-form. Since pre-injury samples of his writing were not readily available, several alternative forms were presented to him, and he indicated the one which he had been most accustomed to use; this form was then used throughout the training programme.

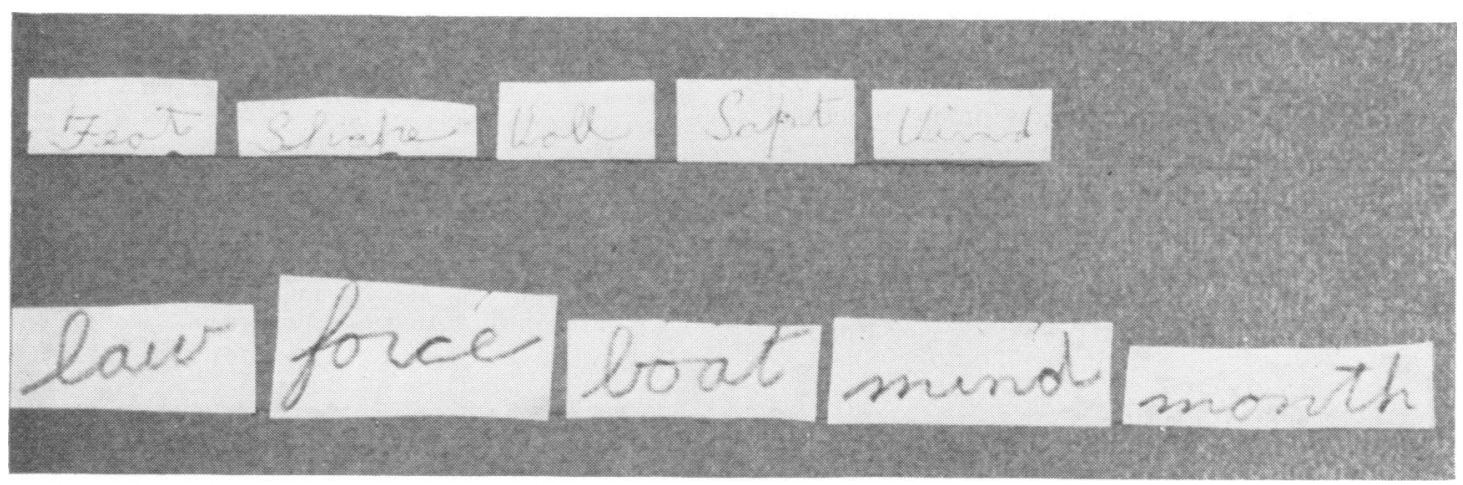

FIG. 2 Changes in writing performance before and after a period of 10 days' training. 
RECODING TECHNIQUE By the use of this technique, the patient's ability to write some letters of the alphabet was built upon. He was asked to consider the letters which he could not write in terms of the ones at which he was more proficient. When, for example, he had to write the letter ' $h$ ', which he found difficult, he was instructed to consider it in terms of the letter ' $n$ ', which he could write well, and then to add a stroke at the side. Similar instructional techniques were based on his proficiency with some upper-case letters. To some extent, the recoding of certain letters in terms of other letters required the learning of simple verbal rules or strategies; in the case of the present patient, care was taken not to make these more difficult than might be compatible with his linguistic ability.

IMPROVEMENT IN WRITING The training represented by the two techniques outlined above was carried out for one to two hours every day for a period of 10 days. A record of the patient's writing performance before and after training was kept, and is indicated in Fig. 2. Initially, the patient's handwriting was very small in size; encouraging the patient to write large forms of the letter made him more aware of the spatial configurations of particular letters.

There are two points which may be made in relation to the improvement which is illustrated in Fig. 2. Firstly, it was interesting to observe, and to be told by the patient, how some letter forms were 'coming back' to him during training - that is, he was able to write certain (previously difficult) letters without the aid of the two techniques outlined above. This occurrence of a form of 'spontaneous recovery' highlights the interaction which can take place between changes in functioning as a result of retraining and spontaneous recovery itself (Humphrey, 1972). The fact that little recovery took place in the six month period between the discharge of the patient and his readmission for cranioplasty suggests that, left to itself, spontaneous recovery did not lead to much improvement in handwriting ability; the subsequent improvement in such a relatively short space of time indicates how much 'potential' for recovery may still remain in the case of an ability which seems to have been severely impaired, and how it can be improved by systematic practice. Secondly, it should be pointed out that the improvement indicated in Fig. 2 refers to that which occurred after 10 days of fairly intensive training. For practical reasons it was not possible to continue this training after the patient left the ward. Consequently, the handwriting of the patient-as tested 15 months after training - had relapsed somewhat. This emphasizes the importance of bringing about a degree of 'overlearning' in the reacquisition of response patterns by brain-damaged patients, and the difficulty in defining the time when such overlearning has occurred. As in other skills, practice is required to establish an automatic pattern.

\section{CONCLUSION}

Precision of concepts and adequacy of experimental control are often difficult to achieve in the field of retraining of psychological functions after brain damage. Consequently, methods of investigation and retraining may not be as generally applicable as in other areas of psychological inquiry. It is possible that a different patient, one who had more insight than our patient (into his problem), might have been able to bring about some improvement in his writing by developing his own strategies for writing individual letters. However, it should be emphasized that it is not so much the particular (relatively simple) methods used in the present case which are of most importance, but rather the general approach of first achieving an accurate understanding of the nature of the patient's difficulty and then, on the basis of this understanding and general principles of retraining, engineering a training programme by which an improvement in function can be brought about.

\section{REFERENCES}

Butfield, E., and Zangwill, O. L. (1946). Re-education in aphasia: a review of 70 cases. Journal of Neurology, Neurosurgery, and Psychiatry, 9, 75-79.

Humphrey, N. (1972). Seeing and nothingness. New Scientist, $53,682-684$.

Luria, A. R., Naydin, V. L., Tsvetkova, L. S., and Vinarskaya, E. N. (1969). Restoration of higher cortical functions following local brain damage. In Handbook of Clinical Neurology, vol. 3, pp. 368-433. Edited by P. J. Vinken and G. W. Bruyn. North-Holland: Amsterdam. 